\title{
Nanofabrication and Electrochemical Characterization of Self-assembled Monolayers sandwiched between Metal Nanoparticles and Electrode Surfaces
}

Pilar Cea*a,b, Santiago Martina,c, Alejandro González-Orive ${ }^{b}$, Henrry M. Osorio ${ }^{a, b}$, Pablo

5 Quintina,b, Lucía Herrera,b

a. Departamento de Química-Física. Universidad de Zaragoza. Spain.

b. Instituto de Nanociencia de Aragón. Laboratorio de Microscopías Avanzadas. Universidad de Zaragoza. Spain.

10 c. Instituto de Ciencia de los Materiales de Aragón. Universidad de Zaragoza-CSIC. Spain. 


\section{ABSTRACT}

Nanoscience and nanotechnology have reached the syllabi of many upper-division

15 undergraduate and master-level courses all over the world. There is therefore a growing need for practical exercises that illustrate the fabrication, characterization, properties and applications of nanomaterials. Here we describe an advanced-level laboratory experiment in which students had the opportunity to fabricate surfaces modified by ordered monolayers and nanostructured materials. The surface modification was

20 quantified by means of a quartz crystal microbalance, whilst the electrochemical properties of the nanoarchitectures were assessed using cyclic voltammetry experiments. Electron transfer across self-assembled monolayers mediated by gold nanoparticles was presented as a topic for discussion, and consideration of potential practical applications of the observed phenomena (catalytic and electrocatalytic

25 processes as well as development of optical, (opto)electronic and photovoltaic devices with enhanced properties) was proposed as a further reading exercise.

\section{ABSTRACT GRAPHIC}

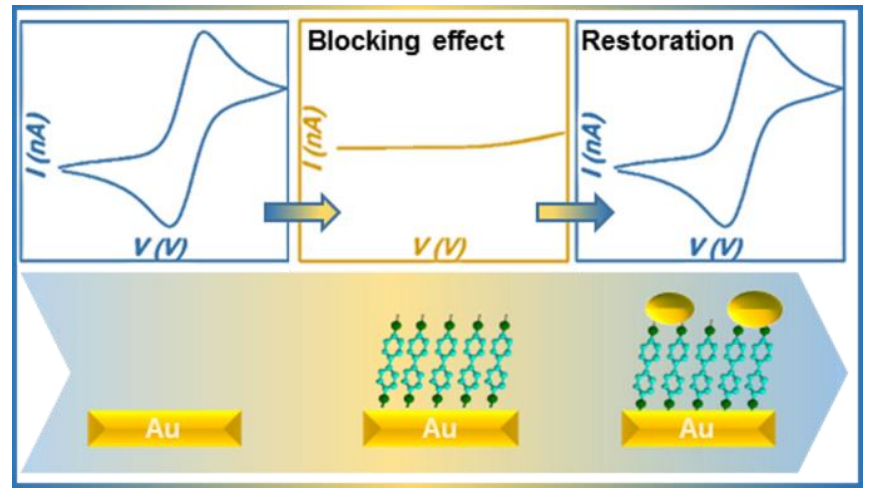

\section{KEYWORDS}

30 Upper-Division Undergraduate, Graduate Education/Research, Multidisciplinary, Hands on learning, Materials Science 


\section{PEDAGOGICAL GOALS}

35 Nanoscience and nanomaterials have found, or been proposed for, applications in many important fields including health, electronics, energy production and storage, purification and environmental cleanup applications, as well as industrial uses (foods, textiles, cosmetics, sports, coatings aerospace and vehicle technologies, catalysis, construction materials, military technology, etc.). This opens the need for designing new

40 laboratory demonstrations ${ }^{1}$ that can illustrate the phenomena occurring at the nanoscale. ${ }^{2-7}$ In this practical exercise, students were guided through several aspects of nanoscience, illustrated through a variety of surface modifications and manipulations. Specifically, the experiment exposed students to the following concepts and skills:

(i) The key concept of 'bottom-up' assembly, illustrated through the formation of a monolayer by means of the self-assembly method.

(ii) Determination of the surface coverage using a quartz crystal microbalance.

(iii) Synthesis of gold nanoparticles by reduction of a metal precursor.

(iv) Immobilize gold nanoparticles onto a thiol terminated monolayer.

(v) The effects of surface coverage illustrated through the cyclic voltammetry response of a redox probe in a bare gold electrode, a gold electrode covered by a tightly-packed monolayer and a gold nanoparticle/organic monolayer/gold sandwich structure.

(vi) The potential applications of the fabricated devices.

\section{EXPERIMENTAL OVERVIEW}

A detailed student handout and the instructor notes for this laboratory experiment are provided in the Supporting Information. Scheme II in the Supporting Information 
summarizes the set of experiments of this practical. The practical was designed to be completed over three laboratory sessions (three hours each), in groups of four or five

60 individuals. A chronogram of the activities carried out is presented in the Supporting Information. The students prepared self-assembled monolayers (SAMs) of a dithiol compound, whose surface coverage was determined by means of a Quartz Crystal Microbalance (QCM). Cyclic voltammetry (CV) experiments demonstrated that the SAM blocked the electron transfer at the electrode. Subsequently, gold nanoparticles (GNPs)

65 were synthetized and deposited onto the SAM surface, which was also quantified by QCM. CV experiments demonstrated that the electron transfer was restored in the GNPs/SAM/gold devices.

\section{EXPERIMENTAL SECTION}

Reagents and Equipment

70 All materials are commercially available and were used as received: biphenyl-4,4'dithiol (95\%) and gold(III) chloride hydrate( 52\% Au basis) were purchased from Aldrich; hexaammineruthenium(III) chloride (ACS reagent, 98\%) and potassium chloride (puriss. p.a. $\geq 99.5 \%$ ) were provided by Sigma and Fluka, respectively. Gold electrodes on glass for the CV studies were purchased from Arrandee. Substrates for the QCM

75 experiments were purchased from Stanford Research (AT-cut, $\alpha$-quartz crystals with a resonant frequency of $5 \mathrm{MHz}$ having circular gold electrodes patterned on both sides). Gold electrodes and QCM substrates can be reused a rather limiting number of times (ca. 3) by cleaning them with piranha solution. Caution: piranha solution reacts violently with most organic materials and may result in explosion or skin burns if not handled with

80 extreme caution. This is the reason why we did not allow our students to use it and our technicians were in charge of cleaning the substrates after the students left the laboratory. Piranha solution was prepared by mixing concentrated sulfuric acid and hydrogen peroxide ( $30 \%$ in volume) in a 3:1 proportion, by adding hydrogen peroxide to 
sulfuric acid very slowly, never in reverse. The substrates were immersed for 5 minutes

85 in a piranha solution and then were thoroughly rinsed with distilled water.

QCM measurements were carried out using a Stanford Research system instrument. CV experiments were done using a potentiostat from Eco Chemie and a standard three electrode cell, where the working electrode was a gold electrode (bare gold, SAM/gold, or GNPs/SAM/gold) connected to the potentiostate by means of a cable ended in a metallic

90 tweezer that held the electrode (see Figure S11 in the Supporting Information), the reference electrode was $\mathrm{Ag} / \mathrm{AgCl}, \mathrm{KCl}(3 \mathrm{M})$ and the counter electrode was a Pt sheet.

\section{HAZARDS}

This practical required the use of ethanol which is a volatile and flammable solvent. Gold(III) chloride hydrate may cause irritation to the skin, eyes, and respiratory tract,

95 and may be harmful if swallowed or inhaled. Sodium borohydride is a toxic, flammable compound and is also a source of basic borate salt, which can be corrosive. There is a flammability hazard associated with the production of hydrogen from the reaction between gold(III) and sodium borohydride. The reaction was performed in a fume hood. Appropriate personal protection equipment (PPE) (gloves, safety glasses or goggles,

100 laboratory coat, covered shoes, etc.) was worn when handling chemical reagents.

\section{EXPERIMENTS AND RESULTS}

Self-assembled films are spontaneously formed on surfaces by chemisorption of the head group of an organic compound onto a substrate. ${ }^{8}$ Typical examples include thiols on gold and trichlorosilanes on glass. The ease of preparation and the fact that it is

105 possible to fabricate SAMs on a variety of substrates using different terminal functional groups have made the self-assembly technique a widely used 'bottom-up' method of surface modification. For further details about SAMs see reference ${ }^{8}$ and the Supporting Information. Figure 1 illustrates the experimental sequence for the formation of a SAM onto a gold electrode. 


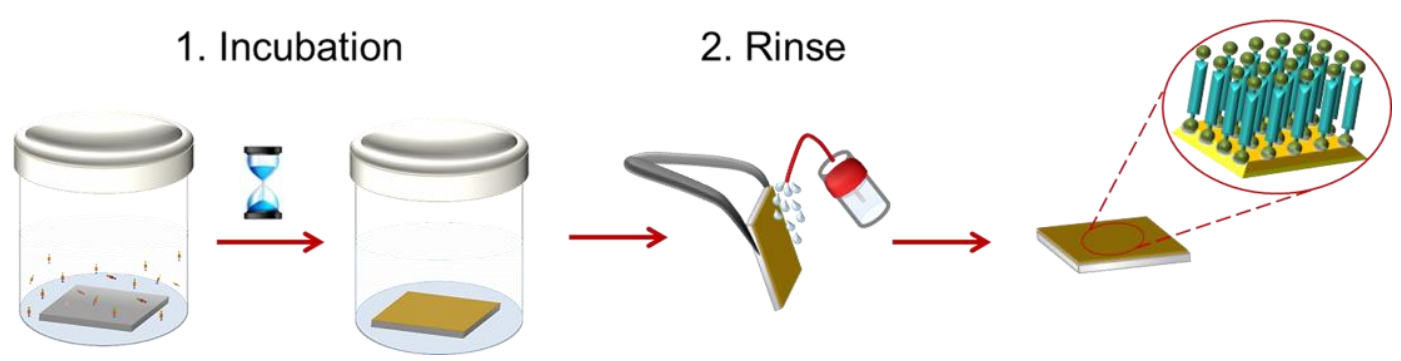

Figure 1. Fabrication of a SAM by immersion of the gold electrode into the solution containing the molecules to be deposited.

Quartz crystal microbalances determine the mass variation per unit area by

115 measuring the change in frequency of a quartz crystal resonator. The QCM can be used under vacuum, in gas phase and in liquid environments. Therefore, it represents a useful tool for the determination of the surface coverage of SAMs onto gold substrates. More information about QCM fundamentals and experiments can be found in reference ${ }^{9}$ and in the Supporting Information.

120 Prior to the SAM formation the resonant frequency of a clean QCM substrate, $f_{0}$, was determined. Afterwards, a gold electrode from Arrandee for the electrochemical experiments and a gold substrate from Standford Research for the QCM studies were introduced in a clean (pre-treated with a $2 \mathrm{M} \mathrm{NaOH}$ solution, rinsed and dried) vessel containing a $1 \mathrm{mM}$ solution of biphenyl-4,4'-dithiol (Figure 2) in absolute ethanol, and 125 incubated at $20^{\circ} \mathrm{C}$ for 24 hours in the absence of light as illustrated in Figure 1.

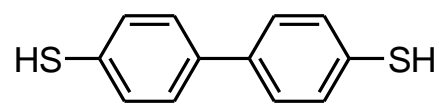

Figure 2. Chemical structure of biphenyl-4,4'-dithiol.

Immersion of the gold electrodes in a biphenyl-4,4'-dithiol solution resulted in the formation of a gold-thiolate monolayer according to: 10

$$
\mathrm{Au}+\mathrm{HS}-\mathrm{R}-\mathrm{SH} \rightarrow \mathrm{Au}-\mathrm{S}-\mathrm{R}-\mathrm{SH}+1 / 2 \mathrm{H}_{2}
$$


The modified gold electrodes were rinsed thoroughly with absolute ethanol to remove any physisorbed material and dried in nitrogen. Afterwards, the resonant frequency of the modified QCM substrate was determined, $f_{1}$ (see cartoon in Figure 3 and Scheme II.b in the Supporting Information). The change in the frequency of oscillation after 24

135 hours of incubation of the QCM substrate in the biphenyl-4,4'-dithiol solution $\left(\Delta f=f_{0^{-}}\right.$ $f_{1}=20 \mathrm{~Hz}$ ) was introduced in the Sauerbrey equation: ${ }^{11}$

$$
\Delta f=-\frac{2 \cdot f_{0}^{2} \cdot \Delta \mathrm{m}}{\mathrm{A} \cdot \rho_{\mathrm{q}}^{1 / 2} \cdot \mu_{\mathrm{q}}^{1 / 2}}
$$

where $f_{0}$ is the fundamental resonant frequency, $\Delta m$ is the mass change, $A$ is the electrode area, $\rho_{\mathrm{q}}$ is the density of the quartz $\left(2.65 \mathrm{~g} \cdot \mathrm{cm}^{-3}\right)$ and $\mu_{\mathrm{q}}$ is the shear module

$140\left(2.95 \cdot 10^{11} \mathrm{dyn}^{\circ} \mathrm{cm}^{-2}\right)$. Figure 3 shows the QCM values at different incubation times. The formation of the monolayer was completed after ca. 24 hours of incubation. The resulting surface coverage for the biphenyl-4,4'-dithiol monolayer on gold was $6.1 \cdot 10^{-10} \mathrm{~mol} \cdot \mathrm{cm}^{-2}$, which is in good agreement with the theoretical value, $6.2 \cdot 10^{-10} \mathrm{~mol} \cdot \mathrm{cm}^{-2}$, determined from a molecular model (Spartan 08 V1.0.0). 


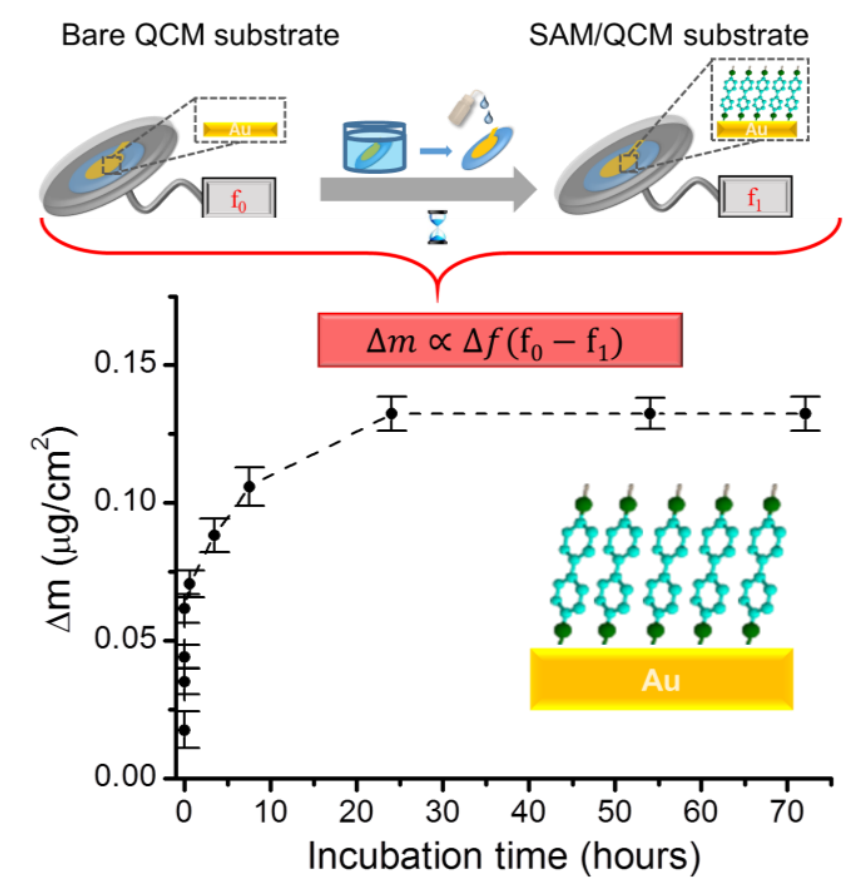

Figure 3. Surface coverage of a gold substrate upon incubation time in a biphenyl-4,4'dithiol solution. Top cartoon: illustration of the experimental procedure for the surface coverage determination.

150 Cyclic voltammetry is a potential sweep method where the potential is changed at a certain scan rate and the current at the working electrode is measured. The plot of current $v$ s. potential is a cyclic voltammogram. $\mathrm{CV}$ is widely used to measure the redox potential of electroactive materials, to determine the electrode reaction mechanisms, and it is also used as an indirect measurement of defect densities in thin films. In this

155 practical, CV was used to study the blocking effect of a tightly-packed monolayer on gold and the electron transfer after deposition of gold nanoparticles onto the monolayer. For ease of understanding and operating the electrochemical section, consult references ${ }^{12-15}$ as well as the Supporting Information. In this practical, a bare gold electrode (working electrode) of $1 \mathrm{~cm}^{2}$ was introduced in an electrochemical cell

160 containing a $1 \mathrm{mM}\left[\mathrm{Ru}\left(\mathrm{NH}_{3}\right)_{6}\right] \mathrm{Cl}_{3}$ and $0.1 \mathrm{M} \mathrm{KCl}$ aqueous solution, the reference electrode was $\mathrm{Ag} / \mathrm{AgCl}, \mathrm{KCl}(3 \mathrm{M})$ and the counter electrode was a Pt sheet (see Figure 
S11 in the Supporting Information). The solution was purged with nitrogen for 30 minutes to remove oxygen, which is electroactive at negative potentials. The scan rate was $0.1 \mathrm{~V} \cdot \mathrm{s}^{-1}$. The electrochemical process that took place during the first half cycle 165 was:

$$
\left[\mathrm{Ru}\left(\mathrm{NH}_{3}\right)_{6}\right]^{3+}+1 \mathrm{e}^{-} \rightarrow\left[\mathrm{Ru}\left(\mathrm{NH}_{3}\right)_{6}\right]^{2+}
$$

The experiment was repeated using the SAM/gold electrode as the working electrode. It is well-known that the deposition of a SAM of an organic material onto an electrode leads to a diminution of the electron transfer to redox species in solution. ${ }^{2,16}$ This

170 phenomenon was clearly observed in the practical (Figure 4). Thus, the lack of Faradaic current confirmed that the SAM was tightly-packed, essentially pinhole free ${ }^{2}$ and effectively blocked electron transfer between the redox probe and the electrode surface. It is also worth mentioning that the blocking effect of the SAM depends largely on the incubation time (inset of Figure 4) indicating a progressive coverage of the gold

175 substrate by the dithiol upon the incubation time till the Faradaic current is completely suppressed after 24 hours of incubation. 


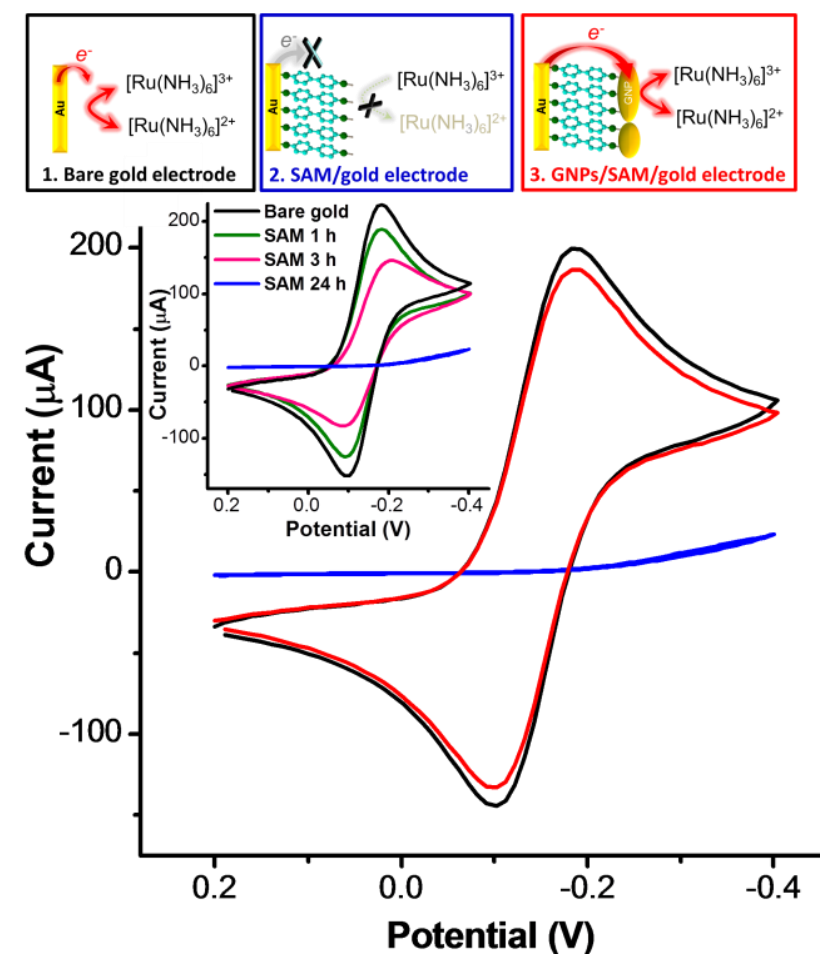

Figure 4. Cyclic voltammograms obtained using a bare gold electrode (black), a gold electrode covered by a biphenyl-4,4'-dithiol SAM (blue), and the SAM covered by GNPs

180 (red) as working electrodes. Inset graph: cyclic voltamograms at the indicated incubation times of the gold electrode in the biphenyl-4,4'-dithiol solution.

A dispersion of gold nanoparticles was prepared by adding rapidly dropwise $0.5 \mathrm{~mL}$ of a $10^{-3} \mathrm{M} \mathrm{NaBH}_{4}$ (freshly prepared) aqueous solution to $30 \mathrm{~mL}$ of a $10^{-5} \mathrm{M} \mathrm{HAuCl}_{4}$ aqueous solution with vigorous stirring at $2{ }^{\circ} \mathrm{C}$ using an ice-water bath. The

185 hydrodynamic diameter of these GNPs was in the 7-28 $\mathrm{nm}$ range. ${ }^{17}$ Incubation of the $\mathrm{SAM} /$ gold substrate in the dispersion of GNPs (at $2{ }^{\circ} \mathrm{C}$ ) started 15 min after the mixture of the reactants. Figure 5 shows the mass change obtained with the QCM versus the incubation time (each point was determined after the substrate was withdrawn from the incubation solution, rinsed with water and dried with nitrogen). An incubation time of

$190100 \mathrm{~min}$ into the GNPs dispersion led to a maximum surface coverage (Figure 5). Additionally, atomic force microscopy (AFM) experiments (Supplemental Information) 
demonstrated the formation of an incomplete gold nanoparticle monolayer on the SAM. These GNPs exhibit a surface coverage of the monolayer around $70 \%$.

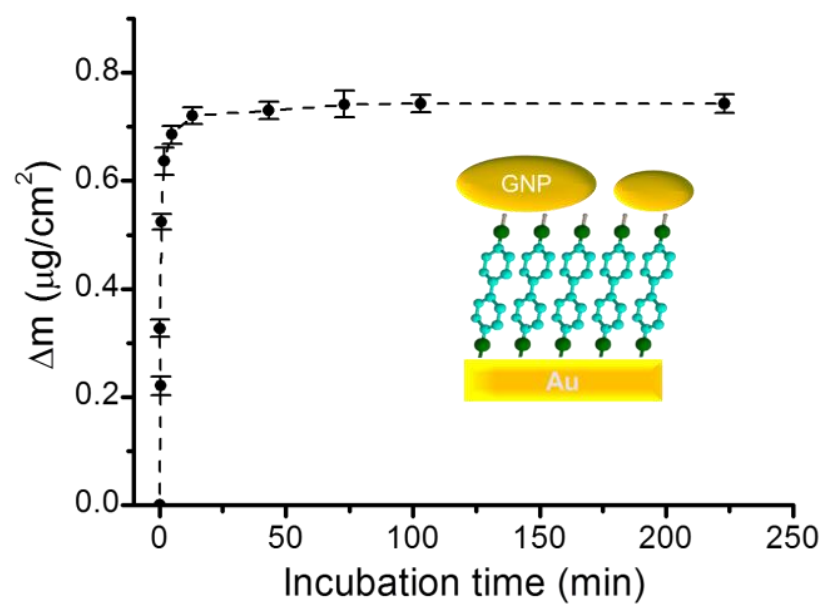

Figure 5. Deposited mass of GNPs $v$ s. incubation time.

Incubation of the SAM/gold devices in a GPNs dispersion did not affect the integrity of the SAM as demonstrated through experiments in the Supporting Information (comparison of the electrodesorption of the thiol as well as gold oxide electroformation

200 before and after the GNPs deposition). After the deposition of the GNPs on the SAM surface, the cyclic voltammogram (Figure 4) exhibited a quasi-reversible behavior with a cathodic-anodic peak separation of $77 \mathrm{mV}$ as well as a near unity peak current ratio as observed for a bare gold electrode under the same conditions. As previously reported, the deposition of conducting nanostructures onto modified electrodes - made of insulating

205 organic layers that completely block the electron transfer when immersed in a redox probe - results in a restoration of the Faradaic current ${ }^{18}$ in systems where disruption of the SAM by penetration of the conducting nanostructures through the layer is ruled out by experimental evidence. ${ }^{19}$ This effect has been observed for several types of conducting nanostructures including metal nanoparticles, ${ }^{19}$ carbon nanotubes, ${ }^{20}$ graphene sheets, ${ }^{21}$

210 quantum dots 22 and even purely molecular objects containing a large number of redox 
centers. ${ }^{23}$ This restoration of the electron transfer has been attributed to a relay station effect of the conducting nanostructures that facilitates the electronic coupling between the underlying electrode and the redox probe. ${ }^{24}$ Therefore, when the conducting nanostructure is attached to the SAM-modified electrode, the electron transfer process

215 involves two consecutive steps: (i) electron transfer between the underlying electrode and the conducting nanostructures, mediated by electron tunneling through the organic layer, and (ii) electron transfer between the conducting nanostructures and the redox probe, which is the rate-limiting step with regard to charge transfer within these particular systems.

\section{EVALUATION AND ASSESSMENT}

The students were asked to answer specific questions prior to the experiment to ensure their understanding of the fundamentals behind this practical (Supporting Information). Through the anwers to these questions the students were expected to attain and

225 demonstrate a strong understanding of the nanofabrication and nanocharacterization methods involved in this practical. Before the commencement of the laboratory sessions students were invited to undertake further reading (Supporting Information) and after completion of the practical entered into a class debate on the potential applications of these nanostructures. In our classes, the instructor initiated the debate by asking why

$230 \mathrm{GNP} / \mathrm{SAM} /$ electrode devices could have interest or applications beyond a conventional electrode. The students in our cohort indicated the different properties of nano-sized gold from the bulk counterpart and also noted that GNPs exhibit a high electrocatalytic activity, which may result in electrocatalytical applications. A significant number of students also highlighted the possibility of forming arrays of electrodes in which each

235 electrode is as small as a few nanometers resulting in a higher sensitivity and selectivity, i.e, sensor applications including biosensors and immunosensors. Some students 
remarked that the passivating organic layer may result in electrodes with a low background capacitance which facilitates low detection limits in electroanalysis. Students also mentioned potential applications in the field of photovoltaics due to the high density

240 of states that can exist on the nanoparticles and also the fact that the energy levels of the nanoparticles are tuneable by altering their size.

Assessment of student understanding of associated concepts was achieved by the laboratory report that students were asked to prepare (Supporting Information). Nearly all

245 students showed a strong grasp on the experiments involved and a good understanding of the practical.

\section{CONCLUSIONS}

This demonstration tied together several nanofabrication and characterization

250 techniques and the observation of new phenomena and properties of materials at the nanoscale. Ultimately, we expect that this comprehensive activity, which builds on the excitement of creating conducting nanostructures with important optical, electronic and catalytic applications, will stimulate interest in nanoscience and nanotechnology and contribute to inspire students to pursue careers in these fields.

\section{ASSOCIATED CONTENT}

Supporting Information

Experimental handout for the students and notes for the instructor are included in the Supporting Information. This material is available via the Internet at

260 http://pubs.acs.org. 


\section{AUTHOR INFORMATION}

Corresponding Author

*E-mail: pilarcea@unizar.es

\section{ACKNOWLEDGMENTS}

We thank students in the Master in Nanostructured Materials for Nanotechnology Applications from Zaragoza University for providing feedback for the improvement of this practical as well as Prof. P. J. Low from the University of Western Australia for useful discussions. Financial support from the Department of Physical Chemistry and 270 DGA/Fondos Feder is acknowledge as well as CTQ2012-33198 and CTQ2013-50187EXP grants.

\section{REFERENCES}

(1) Jones, M. G.; Blonder, R.; Gardner, G. E.; Albe, V.; Falvo, M.; Chevrier, J. Nanotechnology and Nanoscale Science: Educational challenges. International Journal of Science Education 2013, 35, 1490-1512.

(2) Cea, P.; Lopez, M. C.; Martin, S.; Villares, A.; Pera, G.; Giner, I. The use of cyclic voltammetry to probe the passivation of electrode surfaces by well-ordered selfassembly and Langmuir-Blodgett films. J. Chem. Educ. 2009, 86, 723-725.

280 (3) Gottfried, D. S. Review of nanotechnology in undergraduate education. J. Chem. Educ. 2012, 88, 544-545.

(4) Rushton, G. T.; Criswell, B. A. Cutting-edge and cross-cutting: connecting the dots between nanotechnology and high school chemistry. J. Chem. Educ. 2012, 89, 1217-1219.

285 (5) Sekiya, M.; An, A.; Ata, M. Nanocommunication design in graduate-level education and research training programs at Osaka University. J. Nanopart. Res. 2014, 16:2595.

(6) Jones, A. R.; Anderson, A. A.; Yeo, S. K.; Greenberg, A. E.; Brossard, D.; Moore, J. W. Using a deliberative exercise to foster public engagement in nanotechnology. $J$. Chem. Educ. 2014, 91, 179-187.

(7) Bishop, L. M.; Tillman, A. S.; Geiger, F. M.; Haynes, C. L.; Klaper, R. D.; Murphy, C. J.; Orr, G.; Pedersen, J. A.; DeStefano, L.; Hamers, R. J. Enhancing graduate student communication to general audiences through blogging about nanotechnology and sustainability. J. Chem. Educ. 2014, 91, 1600-1605.

295 (8) Ulman, A. An Introduction to Ultrathin Organic Films: From Langmuir-Blodgett to Self-Assembly; Academic Press, Inc.: San Diego, 1991.

(9) Xie, Q.; Li, Z.; Deng, C.; Liu, M.; Zhang, Y.; Ma, M.; Xia, S.; Xiao, X.; Yin, D.; Yao, $\mathrm{S}$. Electrochemical quartz crystal microbalance monitoring of the cyclic 
voltammetric deposition of polyaniline - A laboratory experiments for undergraduates. J. Chem. Educ. 2007, 84, 681-684.

(10) Karpovich, D. S.; Blanchard, G. J. An undergraduate laboratory experiment for the direct measurement of monolayer-formation kinetics. J. Chem. Educ. 1995, 72, 466-470.

(11) Sauerbrey, G. The use of quartz oscillators for weighing thin layers and for microweighing. Z. Physik 1959, 155, 206-222.

(12) Kissinger, P. T.; Heineman, W. R. Cyclic voltammetry. J. Chem. Educ. 1983, 60, 702-706.

(13) Mabbott, G. A. An introduction to cyclic voltammetry. J. Chem. Educ. 1983, 60, 697-702.

310 (14) Van Benschoten, J. J.; Lewis, J. Y.; Heineman, W. R.; Roston, D. A.; Kissinger, P. T. Cyclic voltammetry experiment. J. Chem. Educ. 1983, 60, 772-776.

(15) Bard, A.; Faulkner, L. R. Electrochemical Methods. Wiley, New York, 1980.

(16) Porter, M. D.; Bright, T. B.; Allara, D. L. Spontaneously organized molecular assemblies. 4. Structural characterization of normal-alkyl thiol monolayers on 315 gold by optical ellipsometry, infrared-spectrsocopy, and electrochemistry. J. Am. Chem. Soc. 1987, 109, 3559-3568.

(17) Osorio, H. M.; Cea, P.; Ballesteros, L. M.; Gascon, I.; Marqués-González, S.; Nichols, R. J.; Pérez-Murano, F.; Low, P. J.; Martín, S. Preparation of nascent molecular electronic devices from gold nanoparticles and terminal alkyne functionalised monolayer films. J. Mater. Chem. C. 2014, 2, 7348-7355.

(18) Bethell, D.; Brust, M.; Schiffrin, D. J.; Kiely, C. From monolayers to nanostructured materials: an organic chemist's view of self-assembly. $J$. Electroanal. Chem. 1996, 409, 137-143.

(19) Zhao, J.; Wasem, M.; Bradbury, C. R.; Fermin, D. J. Charge transfer accross selfassembled nanoscale metal-insulator-metal heterostructures. J. Phys. Chem. C. 2008, 112, 7284-7289.

(20) Chou, A.; Eggers, P. K.; Paddon-Row, M. N.; Gooding, J. J. Self-assembled carbon nanotube electrode arrays: effect of length of the linker between nanotubes and electrode. J. Phys. Chem. C 2009, 113, 3203-3211.

330 (21) Xie, X.; Zhao, K.; Xu, X.; Zhao, W.; Liu, S. X.; Zhu, Z.; Li, M.; Shi, Z.; Shao, Y. Study of heterogeneous electron transfer on the graphene/self-assembled monolayer modified gold electrode by electrochemical approaches. J. Phys. Chem. C. 2010, 114, 1423-14250.

(22) Kantor-Uriel, N.; Roy, P.; Saris, S.; Kiran, V.; Waldeck, D. H.; Naaman, R. Evidence for enhanced electron transfer by multiple contacts between selfassembled organic monolayers and semiconductor nanoparticles. J. Phys. Chem. C. 2015, 119, 15839-15845.

(23) Lhenry, S.; Jalkh, J.; Leroux, Y. R.; Ruiz, J.; Ciganda, R.; Astruc, D.; Hapiot, P. Tunneling dendrimers. Enhancing charge transport through insulating layer 340 using redox molecular objects. J. Am. Chem. Soc. 2014, 136, 17950-17953.

(24) Chazalviel, J.-N.; Allongue, P. On the origin of the efficient nanoparticle mediated electron transfer across a self-assembled monolayer. J. Am. Chem. Soc. 2011, 133, $762-764$. 